\title{
Training Future Physicians to Deliver Tobacco Cessation Treatment
}

\author{
Nancy A. Rigotti, MD \\ Tobacco Research and Treatment Center, Division of General Internal Medicine, Department of Medicine, Massachusetts General Hospital and \\ Harvard Medical School, Boston, MA, USA.
}

KEY WORDS: tobacco cessation; medical education.

J Gen Intern Med 31(2):144-6

DOI: $10.1007 / \mathrm{s} 11606-015-3560-7$

(C) Society of General Internal Medicine 2016

$\mathrm{F}$ or two decades already, clinical practice guidelines have exhorted physicians to address tobacco use with all of their adult patients. Evidence from clinical trials was sufficient by 1996 for the U.S. Public Health Service to make this recommendation in the nation's first smoking cessation clinical practice guideline. ${ }^{1}$ Subsequent updates reaffirmed this position. Most recently, the September 2015 update of the U.S. Preventive Services Task Force's tobacco cessation guideline gave this recommendation an " $\mathrm{A}$ " rating. $^{2}$

Translating this guideline into routine practice remains a challenge. Addressing tobacco use - or changing any healthrelated behavior, for that matter-requires doing more than just giving a patient advice to change. Delivering an effective intervention requires a physician to have the skill to help smokers overcome their ambivalence to change, and then to guide them to available and appropriate counseling and pharmacotherapy resources. Because nicotine dependence is a chronic disease, achieving success often requires a sustained effort over time.

The original U.S. tobacco cessation clinical guideline translated the brief physician interventions that had been effective in research settings into five steps, known by the acronym of the "5A's," for use in clinical practice. "Thanks in part to the nudge of Meaningful Use criteria for electronic health records, most physicians, or someone in their practices, now document patients' tobacco use status, thereby achieving the first " $\mathrm{A}$ " (Ask all patients about smoking). Physicians' rates of performing the second step, advising smokers to quit, is also good, if not ideal. ${ }^{3}$ However, physicians' achievement of the three remaining steps (assess readiness to quit, assist smokers to quit, and arrange follow-up) lags. Even at preventive care visits, only $29 \%$ of smokers receive any tobacco cessation assistance, defined as counseling, health education, or a cessation medication prescription. ${ }^{4}$
The ability to deliver these steps is a core physician competency. Learning how to do it requires acquiring skills that have traditionally been poorly taught in medical schools. ${ }^{5}$ An article in this issue reports the results of an ambitious effort to close this curricular gap. Ockene and colleagues developed a comprehensive and innovative multi-modal tobacco education intervention and tested it in a large cluster randomized controlled trial at 10 U.S. medical schools. ${ }^{6}$ At the start, they established that all schools provided fewer than 4 hours total of tobacco education curriculum. The intervention incorporated a variety of state-of-the-art educational methods, and training occurred at more than one point in a student's instruction. One component, designed for first-year students, consisted of a 4-hour web-based course teaching the content of the "5A's", accompanied by a role play demonstration and practice in the classroom setting. This could fit into new "flipped classroom" models of medical education. A second component, designed for the third-year students in their Internal Medicine or Family Medicine clerkship, was a faculty development exercise in which study staff taught clerkship preceptors to model, teach, and provide feedback about tobacco dependence treatment.

To test their intervention, the investigators conducted a rigorous cluster randomized controlled trial that compared medical schools given the intervention to schools that kept their existing curriculum. The goal was improving third-year students' ability to deliver tobacco dependence treatment using the " $5 \mathrm{~A}$ " paradigm. The primary outcome was an objective measure of student skills, a tobacco-specific Objective Structured Clinical Examination (OSCE) case administered to third-year medical students in the Classes of 2012 and 2014. OSCE scores derived from a validated scoring system were compared between intervention and control schools. A secondary outcome was students' self-rated confidence in doing the 5A steps. Students completed a survey twice, in their first and third years, and their scores were compared to those of students in the control schools.

The results were disappointing. There was no significant difference between study arms in the primary outcome, the objective measure of third-year students' scores on the OSCE tobacco case. Although significant differences favoring the intervention schools were observed for five of the composite score's 33 components, there was no adjustment for multiple

Published online January 8, 2016 
testing and the finding is suggestive rather than definitive. A significant difference favoring the intervention schools was observed for three of six components of the secondary outcome measure (students' self-rated ability to provide the Assist and Arrange steps and counsel about pharmacotherapy). The survey findings are consistent with prior work, which shows that a variety of educational methods other than pure didactic lectures can improve student's self-rated tobacco cessation counseling skills and confidence. ${ }^{7}$ However, few studies have assessed students' actual performance of tobacco cessation skills, ${ }^{8}$ as this study did so carefully.

Why didn't the intervention find an effect on objectivelymeasured student performance? I see no flaw in the study design or conduct to explain it. Students in the two study arms were comparable in demographic characteristics, even though randomization occurred at the level of the school. Students participated in the interventions and completed assessments at high rates that differed little by study arm. Careful process measures indicated that the intervention condition was delivered with high fidelity.

One clue is that a substantial number of students in control schools also reported exposure to tobacco-related educational interventions. The group difference in students' report of their duration of exposure to tobacco education over 4 years, measured as number of curriculum hours, had only borderline statistical significance. Perhaps an increase in tobacco education was occurring more broadly, separate from the intervention. Alternately, schools were not blinded to study arm. Schools that were sufficiently interested to participate in this trial might have, intentionally or not, made curricular changes even without access to the special intervention developed for this study.

Another explanation, noted by the authors, is a ceiling effect for some measures. Even the control schools recorded high rates for items measuring the Ask and Advise steps on both the OSCE and the student surveys, making it difficult to demonstrate an intervention effect. In contrast, in the intervention schools, OSCE scores for items that measured the Assist and Arrange steps were relatively low even after the intervention. Perhaps the multi-modal intervention, while comprehensive, was not strong enough. The authors suggest that not having a curricular component for second-year students might have attenuated the intervention's overall effect.

Alternately, student performance on a single OSCE case might have been too blunt a tool or have set too high a bar for measuring student's abilities. Students were assessed on 33 different actions in the OSCE. This is perhaps a more detailed intervention than is realistic for today's busy practice environment or for the limited time permitted in the OSCE. Abbreviated versions of the " $5 \mathrm{~A}$ 's" consisting of two or three steps are already developed. As primary care practice moves toward team-based care, some of the $5 \mathrm{~A}$ steps will likely be done by other members of the care team. While physicians should still be capable of the full " $5 \mathrm{~A}$ " intervention when the opportunity arises to provide it, expecting a full demonstration by thirdyear students might not be realistic.

Overall, the study by Ockene and colleagues is an outstanding example of high-quality medical education research. Using state-of-the-art methods, the investigators changed the curriculum at five medical schools and conducted a careful randomized controlled trial to determine whether their intervention made a difference. While the study did not unequivocally prove the intervention's effectiveness, the totality of the findings suggest to me that the approach is on the right track. Future work should aim to strengthen the intervention. Eventually, this approach might be broadened to teach students about changing health-related behavior in general. In the meantime, interventions like the ones tested in this study are valuable models for curriculum committees in U.S. medical schools to consider adopting now.

Ultimately, building adequate tobacco cessation education into medical school curricula nationwide will require more than just defining an effective program. Space in a medical school curriculum is a precious commodity. Creating room for new topics or new material, no matter how important or wellvalidated, is always a challenge. Advocates for increasing medical schools' teaching of skills to address tobacco dependence-or to change unhealthy behavior in general-face this barrier. One important influence on curricular decisions is what topics are covered on the U.S. Medical Licensing Examinations (USMLE). ${ }^{9}$ Here, there is a glimmer of hope. In 2012, the New York State Tobacco Education Task Force formally recommended to the National Board of Medical Examiners (NBME) that tobacco dependence and treatment content be included in each of the three USMLE tests. ${ }^{9}$ The NBME accepted the recommendation in 2013, and content is being developed. It could appear on examinations that will be fielded after 2016. This policy change has the potential to give medical schools a new incentive to enhance their coverage of tobacco dependence education. If so, studies like the one in this issue provide valuable models to consider. Its findings were not definitive but until better programs are identified, the one developed for this study provides a valuable starting point.

Corresponding Author: Nancy A. Rigotti, MD; Tobacco Research and Treatment Center, Division of General Internal Medicine, Department of MedicineMassachusetts General Hospital and Harvard Medical School, Boston, MA, USA (e-mail: nrigotti@partners.org).

\section{Compliance with Ethical Standards:}

Conflict of Interest: The author declares that she does not have a conflict of interest. 


\section{REFERENCES}

1. Fiore MC, Bailey WC, Cohen SJ, et al. Smoking cessation. Clinical Practice Guideline No. 18. Rockville, MD, U.S. Department of Health and Human Services, Public Health Service, Agency for Health Care Policy and Research. AHCPR Publication No. 96-0692. April 1996.

2. Siu AL, for the U.S. Preventive Services Task Force. Behavioral and pharmacotherapy interventions for tobacco smoking cessation in adults, including pregnant women: U.S. Preventive Services Task Force Recommendation. Ann Intern Med. 2015;163(8):622-34. doi: 10.7326/M15-2023.

3. Guinn VP, Hollis JF, Smith KS, Rigotti NA, Solberg LI, Hu W, Stevens VJ. Effectiveness of the 5-As tobacco cessation treatments in nine HMOs. J Gen Intern Med. 2009;24: 149-54.

4. Jamal A, Dube SR, Babb SD, Malarcher AM. Tobacco use screening and cessation assistance during physician office visits among persons aged 1121 years-National Ambulatory Medical Care Survey, United States, 20042010. MMWR. 2014;63:71-9.
5. Ferry LH, Grissino LM, Runfola PS. Tobacco dependence curricula in US undergraduate medical education. JAMA. 1999;282(9):825-9.

6. Ockene JK, Hayes RB, Churchill LC, Crawford SL, Jolicoeur DG, Murray DM, Shoben AB, David SP, Ferguson KJ, Huggett KN, Adams M, Okuliar CA, Gross RL, Bass PF 3rd, Greenberg RB, Leone FT, Okuyemi KS, Rudy DW, Waugh JB, Geller AC. Teaching medical students to help patients quit smoking: outcomes of a 10-school randomized controlled trial. J Gen Intern Med. 2015. doi:10.1007/s11606-015-3508-y.

7. Spangler JG, George G, Foley KL, Crandall SJ. Tobacco intervention training: current efforts and gaps in US medical schools. JAMA. 2002;288(9):1102-9.

8. Stolz D, Langewitz W, Meyer A, Pierer K, Tschudi P, S'ng CT, Strobel W, Perruchoud AP, Fagerström K, Tamm M. Enhanced didactic methods of smoking cessation training for medical students-a randomized study. Nicotine Tob Res. 2012;14(2):224-8.

9. Folan PA, Juster HR, Lennon SE, Briest PJ, Gero CB. Improving tobacco dependence treatment delivery: medical student training and assessment. Am J Prev Med. 2015 (in press). doi:10.1016/j.ampre.2015.04.005. 